Supporting Information

\title{
Analysis of tertiary structural features of branched DNA nanostructures with partially common sequences using small-angle X-ray scattering
}

Mengmeng Tan ${ }^{1}$, Natsuki Takahashi ${ }^{1}$, Shota Fujii ${ }^{2}$, Kazuo Sakurai ${ }^{2,3}$, Kosuke Kusamori', Yuki Takahashi ${ }^{1}$, Yoshinobu Takakura ${ }^{1}$ and Makiya Nishikawa ${ }^{1,4, *}$

1 Department of Biopharmaceutics and Drug Metabolism, Graduate School of Pharmaceutical Sciences, Kyoto University, 46-29, Yoshidashimoadachi-cho, Sakyoku, Kyoto 606-8501, Japan.

${ }^{2}$ Department of Chemistry and Biochemistry, University of Kitakyushu, 1-1 Hibikino, Wakamatsu-ku, Kitakyushu, Fukuoka 808-0135, Japan.

${ }^{3}$ Structural Materials Science Laboratory SPring-8 Center, RIKEN Harima Institute Research 1-1-1 Kouto, Sayo-cho, Sayo, Hyogo 679-5148, Japan.

4 Laboratory of Biopharmaceutics, Faculty of Pharmaceutical Sciences, Tokyo University of Science, 2641 Yamazaki, Noda, Chiba 278-8510, Japan.

*Corresponding Author E-mail: makiya@rs.tus.ac.jp 
Table S1. The sequences of ODNs used for Tetrapodna preparations. REAGENT or RESOURCE

\begin{tabular}{|c|c|}
\hline Tet(sym)-01* & GCTAGACGTTCGATACCT AGCTAAGGTAGCTCTAGC \\
\hline Tet(sym)-02 & GCTAGAGCTACCTTAGCT TCCATACGTTGCAGAACC \\
\hline Tet(sym)-03 & GGTTCTGCAACGTATGGA TGCATTGCATCGAGATGC \\
\hline Tet(sym)-04 & GCATCTCGATGCAATGCA AGGTATCGAACGTCTAGC \\
\hline Tet(id8)-01 & GCTAGACGTTCGATAGCA TGCTAAGGTAGCTCTAGC \\
\hline Tet(id8)-02 & GCTAGAGCTACCTTAGCA TGCTTACGTTGCAGAACC \\
\hline Tet(id8)-03 & GGTTCTGCAACGTAAGCA TGCTTTGCATCGAGATGC \\
\hline Tet(id8)-04 & GCATCTCGATGCAAAGCA TGCTATCGAACGTCTAGC \\
\hline Tet(id12)-01 & GCTAGACGTTCGATAGCA TGCTATGGTAGCTCTAGC \\
\hline Tet(id12)-02 & GCTAGAGCTACCATAGCA TGCTATCGTTGCAGAACC \\
\hline Tet(id12)-03 & GGTTCTGCAACGATAGCA TGCTATGCATCGAGATGC \\
\hline Tet(id12)-04 & GCATCTCGATGCATAGCA TGCTATCGAACGTCTAGC \\
\hline Tet(id16)-01 & GCTAGACGTTCGATAGCA TGCTATCGTAGCTCTAGC \\
\hline Tet(id16)-02 & GCTAGAGCTACGATAGCA TGCTATCGTTGCAGAACC \\
\hline Tet(id16)-03 & GGTTCTGCAACGATAGCA TGCTATCGATCGAGATGC \\
\hline Tet(id16)-04 & GCATCTCGATCGATAGCA TGCTATCGAACGTCTAGC \\
\hline Tet(asym12)-01* & GCTAGACGTTCGATACCTAGCTAA GGTAGCTCTAGC \\
\hline Tet(asym12)-02 & GCTAGAGCTACC AATGCATCCATACGTTGCAGAACC \\
\hline Tet(asym12)-03 & GGTTCTGCAACGTATGGATGCATT CCATCGAGATGC \\
\hline Tet(asym12)-04 & GCATCTCGATGG TTAGCTAGGTATCGAACGTCTAGC \\
\hline Tet(asym16)-01* & GCTAGACGTTCGATACCTAGCTAAGG TAGCTCTAGC \\
\hline Tet(asym16)-02 & GCTAGAGCTA GCAATGCATCCATACGTTGCAGAACC \\
\hline Tet(asym16)-03 & GGTTCTGCAACGTATGGATGCATTGC ATCGAGATGC \\
\hline Tet(asym16)-04 & GCATCTCGAT CCTTAGCTAGGTATCGAACGTCTAGC \\
\hline
\end{tabular}


Figure S1. SAXS profiles of experimental data and theoretical curves using the rigid four-rod model.

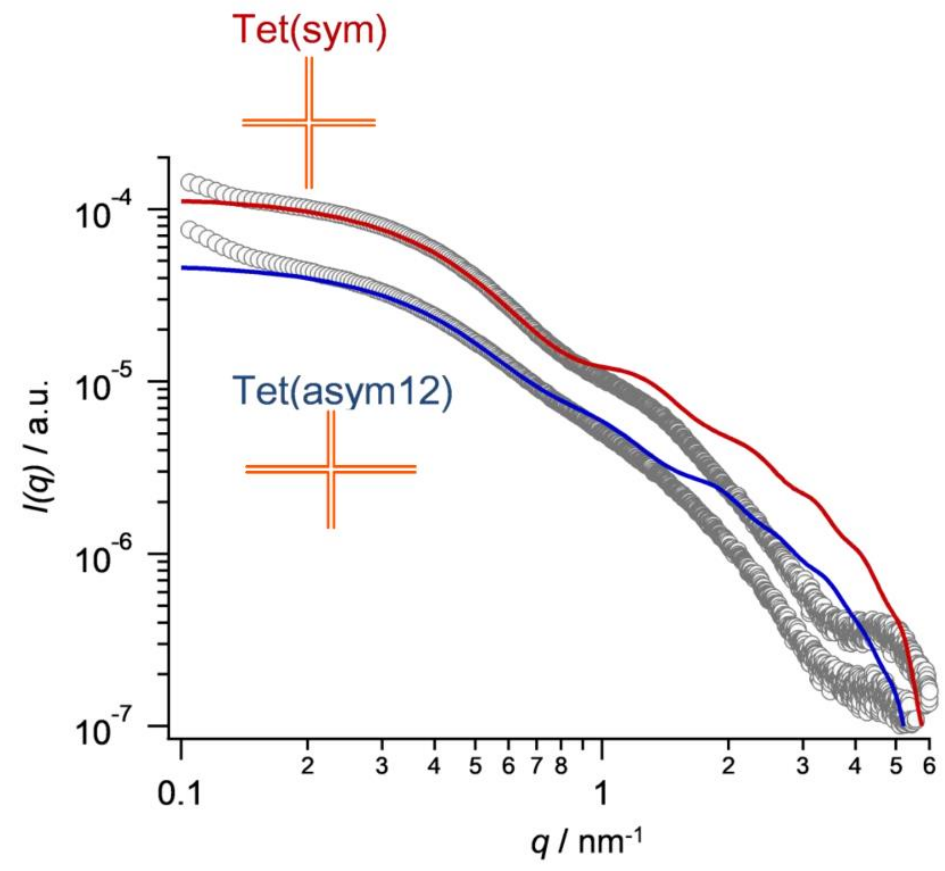

